\title{
Anuloplastia sem suporte para tratamento da insuficiência mitral reumática
}

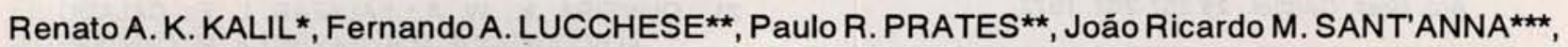
Farid C. FAES ${ }^{\star \star}$, Edemar M. C. PEREIRA**, Ivo A. NESRALLA*

KALIL, R. A. K.; LUCCHESE, F. A.; PRATES, P. R.; SANT'ANNA, J. R. M.; FAES, F. C.; PEREIRA, E. M. C.; NESRALLA, I. A. - Anuloplastia sem suporte para tratamento da insuficiência mitral reumática. Rev. Bras. Cir. Cardiovasc., 7(3):186-193, 1992.

RESUMO: Desde 1974, temos empregado anuloplastia sem suporte para tratamento da insuficiência mitral de etiologia reumática em uma população predominantemente jovem. A avaliaçāo dos resultados tardios forma a base deste relato. Sảo 154 pacientes com insuficiência mitral isolada (sem estenose ou dupla lesāo), sendo $55(36 \%)$ do sexo masculino e $99(64 \%)$ do feminino, idade média $=36 \pm 16$ (5 a 73 ) anos; as lesōes associadas foram: 47 lesōes aórticas, 21 tricuspídeas e 2 comunicaçōes interatriais; a classe funcional pré-operatória foi I-II em $19 \%$ e III-IV em $81 \%$ e o índice cardiotorácico $0,61 \pm 0,10$. Todos os pacientes foram submetidos a uma anuloplastia similar à descrita por WOOLER et alii ${ }^{21}$ que consiste na reduçāo da porção mural do anel mitral obtida pela aplicação de dois pontos ancorados em feltros nas comissuras, sem comprometer a extensão de cúspide septal. Quando necessário, procedimentos adicionais sobre as cordas tendíneas foram realizados. Nenhum paciente recebeu suporte anular por anel ou barra posterior. A mortalidade precoce foi de $3(1,9 \%)$ pacientes: falência miocárdica (1) e embolia pulmonar (2). A mortalidade tardia foi de $9(5,8 \%)$, falência miocárdica (3), septicemia (1), embolia pulmonar (1) e morte súbita (1) e causa desconhecida (3). Reoperaçōes foram realizadas em $28(18,2 \%)$ casos, dos quais $2(1,3 \%)$ por disfunçāo de prótese äórtica. Sopro sistólico residual esteve presente em $48 \%$ dos casos. Complicaçōes tardias: embolias sistêmicas $5,8 \%$ (1/3 como prótese aórtica), endocardite infecciosa $1,3 \%$ e embolia pulmonar $0,7 \%$. Classe funcional pós-operatória (p. a.) foi I - II em $84 \%$ e III - IV em $16 \%$; índice cardiotorácico p. $0.0,58 \pm 0,10$. Probalidade atuarial de sobrevida tardia é estimada em $79,5 \pm 5,3 \%$ aos 10 anos e $71,0 \pm$ $7,4 \%$ aos 14 anos. Sobrevida sem eventos: $67,9 \pm 8,9 \%$ aos 10 anos e $56,1 \pm 11,7 \%$ aos 14 anos. A insuficiência mitral reumática pode ser tratada efetivamente por anuloplastia sem suporte anular protético, com resultados tardios comparáveis àqueles obtidos por técnicas mais complexas. Isto tem importância no tratamento de crianças e adultos jovens, especialmente no sexo feminino, quando se deseja evitar implante de próteses mecânicas. cirurgia.

DESCRITORES: valva mitral, cirurgia; valvoplastia; insuficiência mitral reumática; valvas cardíacas,

\section{INTRODUÇĀO}

A valvoplastia parece ter sido estabelecida como o procedimento de escolha para correção da insuficiência mitral. Na maior parte das vezes, têm sido empregados anéis rígidos ou flexíveis, ou ainda, barras de suporte do anel para remodelamento da estrutura valvar ${ }^{1,4-6,10}$. Na maioria das séries publicadas, há predomínio de lesões não-reumáticas da valva

\footnotetext{
Trabalho realizado na Instituto de Cardiologia do Rio Grande do Sul e Fundaçảo Universitária de Cardiologia. Porto Alegre, RS, Brasil. Apresentado ao $19^{\circ}$ Congresso Nacional de Cirurgia Cardíaca, Sâo Paulo, 7 a 9 de maio, 1992.

* Do Instituto de Cardiologia do Rio Grande do Sul e Fundaçāo Universitária de Cardiologia.

* Do Instituto de Cardiologia.

* Da Universidade Federal do Rio Grande do Sul.

Endereço para separatas: Renato Kalil. Av. Princesa Isabel, 397. 90620 Porto Alegre, RS, Brasil.
} 
mitral 7. 9. 10. 15. Prévios resultados na insuficiência mitral reumática têm sido menos favoráveis ${ }^{\circ} \mathrm{ou}$ apresentam pouco seguimento '.

Desde 1974, temos empregado uma técnica de anuloplastia sem suporte, semelhante à descrita por WOOLER et alii. ${ }^{21}$, em 1962, para correção de insuficiência mitral de qualquer etiologia.

Neste trabalho, apresentamos os resultados a longo prazo do grupo de etiologia reumática.

\section{CASUÍSTICA E MÉTODOS}

Entre 1974 e 1991, foram operados 154 pacientes para correção da insuficiência mitral reumática. Eram $55(38 \%)$ do sexo masculino e $99(66 \%)$ do feminino. A idade variou de 5 a 73 anos (média $36 \pm$ 16 anos). Casos de outras etiologias que nāo a reumática não estão incluídos nesta série. Havia 90 pacientes com lesōes associadas, como segue: 47 lesões aórticas, 21 insuficiências tricúspides e 2 comunicaçōes interatriais. Classe funcional pré-operatória era l ou ll em 29 (19\%) casos e III ou IV em 125 $(81 \%)$; o índice cardiotorácico aos raios $X$ no préoperatório era $0,61 \pm 0,10$. Todos os pacientes foram submetidos ao procedimento, como ilustrado na $\mathrm{Fi}$ gura 1, que consistiu na reduçāo da porção mural do anel valvar, obtida pela aplicação de pontos "em U", de Poliéster 2-0, ancorados em feltros de Teflon eim cada comissura, de maneira a obter reduçāo da circunferência do anel, sem comprometer a extensāo da cúspide septal. Este procedimento é similar ao descrito por KAY e EGERTON ${ }^{14}$, REED et alii ${ }^{20} \mathrm{e}$ WOOLER et alii ${ }^{21}$. Quando necessário, procedimen-

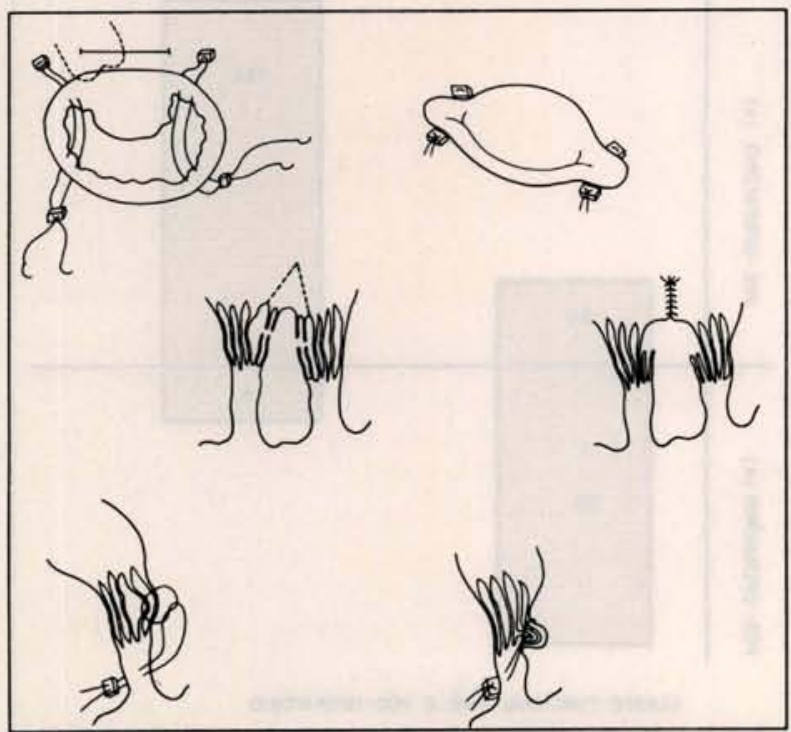

Fig. 1 tos adicionais foram realizados nas cúspides ou nas cordas tendíneas em casos de alongamento ou rotura. No caso de alongamentos, a técnica empregada consistiu na sutura da corda comprometida na altura considerada adequada, baixando-a e fixando-a ao respectivo músculo papilar com Poliester 2-0 ancorado em feltro de Teflon na face oposta do mesmo. Esse encurtamento da corda foi melhor realizado antes da anuloplastia. No caso de dúvida quanto à necessidade de encurtamento de cordas, os pontos comissurais foram aproximados com torniquetes de borracha (os mesmos utilizados para as suturas em bolsa das cânulas) e a valva testada com solução salina; com a valva sem refluxo, os pontos eram fixados. Caso contrário, os torniquetes eram afrouxados e o aparelho subvalvar submetido a avaliação e correção. Para rotura de cordas da cúspide anterior, foi realizada ressecção triangular do segmento sem sustentação ${ }^{16} \mathrm{e}$, para rotura de cordas da cúspide posterior, realizamos ressecção quadrangular ${ }^{5,17}$.

No caso de rotura da cús pide anterior, sempre foi necessária anuloplastia nas comissuras, o que, em geral, năo ocorria após correção da cúspide posterior.

Os pacientes foram operados com esternotomia mediana, sob circulação extracorpórea convencional, canulação das veias cavas e aorta, e emprego de oxigenador de bolhas, induzindo hipotermia moderada a $28^{\circ}-30^{\circ} \mathrm{C}$. Incisāo atrial esquerda após o pinçamento da aorta. Para proteção miocárdica, foi empregada cardioplegia cristalóide hipercalêmica e resfriamento da cavidade pericárdica com solução salina a $4^{\circ} \mathrm{C}$, exceto nos caso operados até 1977 , quando apenas resfriamento externo do coração era realizado. As lesōes cardíacas associadas eram corrigidas apropriadamente, após a valvoplastia mitral. Os tempos médios de perfusão extracorpórea, em toda a série, foi $48 \pm 19 \mathrm{~min}$ e de $40 \pm 11 \mathrm{~min}$ nos casos sem lesōes associadas. Os tempos de anóxia miocárdica foram: $29 \pm 16 \mathrm{~min}$ para toda a série e $23 \pm 8 \mathrm{~min}$ para a valvoplastia isoladamente. Testes de suficiência valvar foram realizados com solução salina gelada infundida na cavidade ventricular por meio de seringa de borracha, através da valva mitral, enquanto na vigência da cardioplegia. Em alguns casos, pequenos jatos de regurgitação foram tolerados, quando procedimentos adicionais pudessem comprometer 0 diâmetro do orifício valvar, causando estenose e os inconvenientes de uma prótese artificial. Com o desenvolver da experiência e o maior entendimento da participaçāo do aparelho subvalvar no mecanismo da regurgitação, tem sido mais freqüente o emprego de encurtamento de cordas e os casos de insuficiência residual são mais raros.

No pós-operatório, foram tomados os cuidados de rotina após cirurgia cardíaca com circulação extracorpórea. Cuidados especiais foram tomados 
KALIL, R. A. K.; LUCCHESE, F. A.; PRATES, P. R.; SANT'ANNA, J. R. M.; FAES, F. C.; PEREIRA, E. M. C.; NESRALLA, I. A. Anuloplastia sem suporte para tratamento da insuficiência mitral reumática. Rev. Bras. Cir. Cardiovasc., 7(3):186-193, 1992.

para detecçāo e tratamento precoces de surtos de reagudizaçāo da febre reumática, incluindo-se terapêutica intensiva com anti-inflamatórios e corticosteróides. Taquicardias sem causa aparente no pósoperatório imediato eram inicialmente tratadas como de origem reumática, enquanto os testes de laboratório eram processados, isso com a finalidade de evitar qualquer enfraquecimento inflamatório do tecido valvar que pudesse causar rotura das suturas.

Para análise clínica, os pacientes foram tabulados de acordo com idade, sexo, lesāo cardíaca associada, procedimentos associados, classe funcional (NYHA) e presença de sopro de regurgitaçāo no pósoperatório. Foi medido o índice cardiotorácico. Ecocardiogramas não foram realizados de rotina em todos os pacientes, bem como cateterismo cardíaco. Estudo ecodopplercadiográfico foi realizado preferencialmente para avaliação e quantificaçāo de regurgitaçāo residual quando presente, bem como para verificaçāo da funçāo ventricular esquerda. A ocorrência de óbitos nos primeiros 30 dias foi considerada mortalidade imediata. Os pacientes foram seguidos pessoalmente pelo cirurgiāo, ou no ambulatório do hospital, ou por seu médico assistente e destes, os dados foram obtidos através de telefone ou carta. Sobrevida sem eventos foi definida como a probabilidade de o paciente estar vivo e livre de reoperação ou complicação maior relacionada à valva na época da avaliação. Pacientes reoperados por outras causas (ex. disfunção de prótese aórtica) embora nāo constituam falências do procedimento mitral, foram incluídos nas reoperaçōes para fins estatísticos. Os resultados foram expressos como média \pm erro-padrảo. Probabilidade de sobrevida foi calculada pelo método atuarial.

\section{RESULTADOS}

Ocorreram três $(1,9 \%)$ óbitos imediatos, devidos a falência miocárdica em um e embolia pulmonar em dois. A mortalidade tardia foi $9(5,8 \%)$ casos e suas causas foram: falência miocárdica $3(1,9 \%)$, septicemia $1(0,6 \%)$, embolia pulmonar $1(0,6 \%)$, morte súbita $1(0,6 \%)$ e causa desconhecida $3(1,9 \%)$ (Tabela 1)

Trinta pacientes foram reoperados em um tempo médio de seguimento de 69 meses ( 5 anos e 9 meses), de 2 meses a 10 anos de pós-operatório. Disfunção de prótese aórtica foi a causa de $2(1,3 \%)$ reoperaçōes, estenose mitral em $2(1,3 \%)$ e insuficiência mitral em 26 (16,9\%). Em duas ocasiōes foi refeita a anuloplastia na reoperaçāo. (Tabela 2).

Complicaçōes tardias foram: embolia sistêmica 9 $(5,8 \%)$ casos, 3 dos quais portadores de prótese aórtica, endocardite infecciosa em $2(1,3 \%)$ e embolia pulmonar em $1(0,6 \%)$. Como nảo houve o emprego rotineiro de anticoagulantes, nāo ocorreram complicaçōes nesse setor (Tabela 3).

$\mathrm{Na}$ avaliação tardia, de 113 sobreviventes sem reoperaçōes, havia 95 ( $84 \%$ ) em classe funcionall ou II e $18(16 \%)$ em classe III ou IV (Figura 2$)$. O índice cardiotorácico pós-operatório foi $0,58 \pm 0,10$. Sopro sistólico de regurgitaçāo residual esteve presente em $48 \%$ dos sobreviventes.

A sobrevida tardia atuarial foi $89,3 \%$ aos 5 anos, $79,5 \%$ aos 10 anos e $71,0 \%$ aos 14 anos, incluindo a mortalidade imediata (Figura 3 ). A sobrevida livre de eventos foi $86,0 \%$ aos 5 anos $67,9 \%$ aos 10 anos e $56,1 \%$ aos 14 anos (Figura 4 ).

\section{COMENTÁRIOS}

A vavoplastia constitui hoje o método preferencial para tratamento de insuficiência mitral ${ }^{3}$. Muitas séries publicadas incluem casos predominantemente de etiologia degenerativa. Quase sempre, alguma forma de suporte com material sintético, seja anel ou barra posterior é implantado ${ }^{4-6}$. Implante de anéis tem sido justificado sob o conceito de remodelamento do anel, julgado por alguns como sendo de importância na manutenção de um reparo adequado e durável ${ }^{9}$. 10, 15. Poucos relatos se referem a anuloplastia sem suporte $2,11-13,18,19$.

A insuficiência mitral reumática tem sido considerada com poucas possibilidades de recuperaçāo valvar e a maior freqüência de eventos relacionados

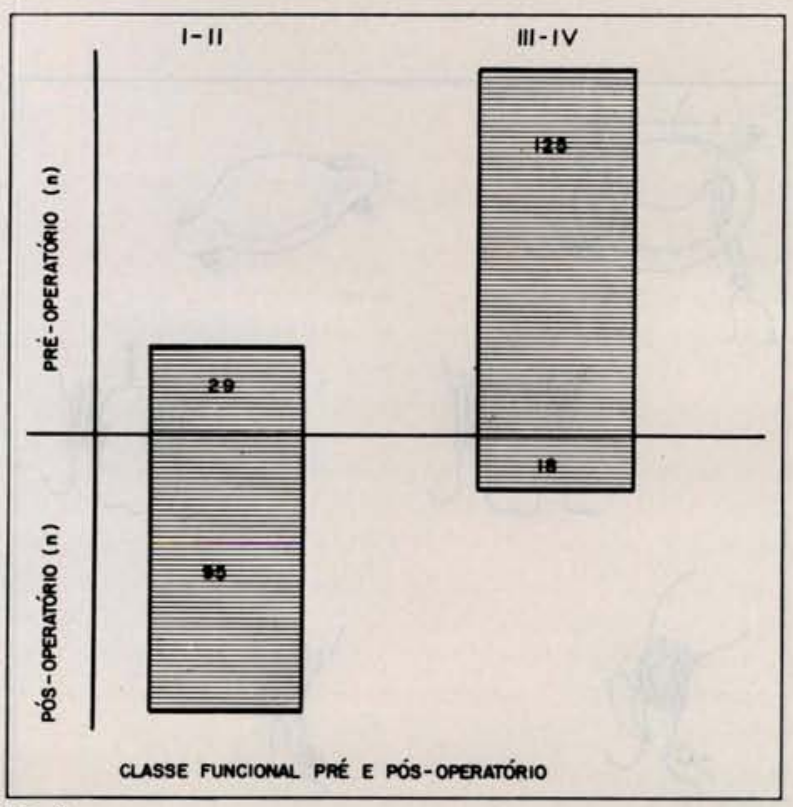

Fig. 2 


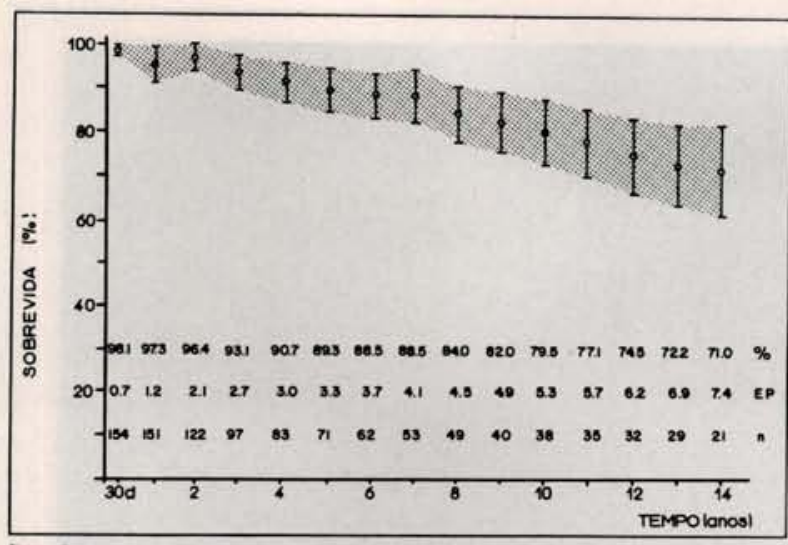

Fig 3

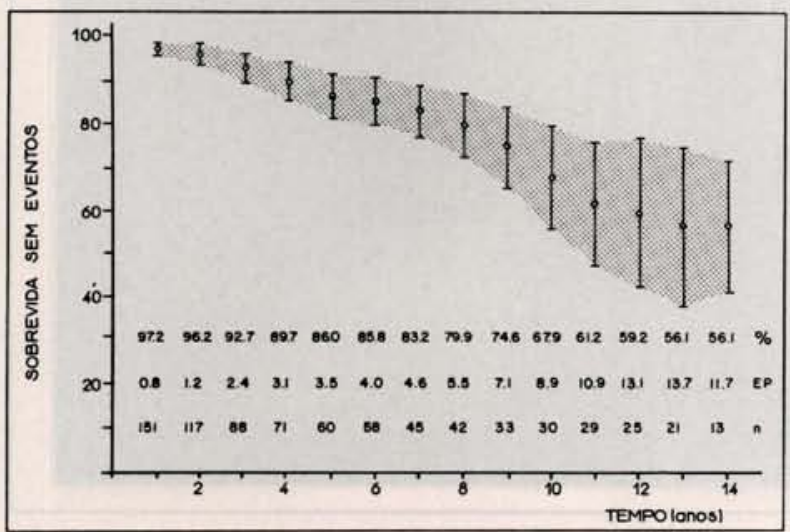

Fig. 4

à valva corrigida ', embora relacionada a maior sobrevida ${ }^{15}$.

Os pacientes incluídos neste trabalho apresentavam regurgitação mitral como lesão predominante. Alguns tinham lesōes associadas, mas nenhumestenose mitral. Os anéis estavam invariavelmente dilatados, as cúspides em calcificaçōes ou fusōes ao nível da cordoalha tendinosa. Fibrose difusa leve a moderada foi o achado característico, associado algumas vezes a rotura ou alongamento de cordas. Os resultados a longo prazo na série inicial de 50 casos operados foram previamente publicados ${ }^{12,13}$.

Este método de reparo valvar mitral respeita basicamente os mesmos princípios já divulgados por KAY \& EGERTON ${ }^{14}$, REED et alii. ${ }^{20} \mathrm{e}$ WOOLER et alii ${ }^{21} \mathrm{e}$ incorpora intervençōes sobre a cordoalha similares às descritas por McGOON ${ }^{16}$. NUNLEY \& STARR ${ }^{17} \mathrm{e}$ CARPENTIER et alii $^{5}$. As diferenças residem no encurtamento anular conseguido por pontos isolados ancorados em feltros de Teflon, sem medição padronizada, colocados de maneira tal que a extensão da cúspide septal é totalmente preservada e ambas são forçadas a como que invaginar para a cavidade ventricular à medida que as suturas sảo fixadas. 0 encurtamento das cordas é obtido de maneira muito simples e a necessidade de realizá-lo é avaliada após colocação temporária de torniquetes de borracha, facilitando a avaliação e melhorando os resultados finais.

Nos casos de doença degenerativa, as suturas podem não ser suportadas como nas etiologias reumática e congênita. $\mathrm{Na}$ doença mixomatosa, poderia ser aconselhável suturar uma barra de feltro de Teflon ou de pericárdio bovino, à porçāo posterior do anel, entre os pontos comissurais, como tem sido empregada por FANTINI $\left(^{\star}\right)$ e, algumas vezes, por nós. As características friáveis do tecido mixomatoso poderia ser a razāo da preferência, pela maioria dos autores, do implante de anéis de suporte. Baseados nos resultados aqui apresentados e comparando-os com séries de pacientes reumáticos que tiveram anéis implantados ${ }^{1.9 .15}$, pode-se inferir que, para a insuficiência mitral reumática, uma simples anuloplastia tipo Wooler, sem suporte anular, é suficiente e mantém resultado similar a longo prazo. Nos casos de reumáticos, quando são apresentados em separado, LESSANA et alii ${ }^{15}$ apresentam $67 \%$ de sobrevida sem eventos aos 13 anos e CARPENTIER et alii ${ }^{5}$ $51 \%$. Nossa série (Figura 3 ) mostra $56,1 \%$ de sobrevida sem eventos aos 14 anos de seguimento.

As causas de mortalidade imediata nāo foram relacionadas à valva, da mesma forma que a mortalidade tardia. Isto pode ser devido à lenta deterioraçāo do reparo, quando ocorre, permitindo tempo para nova investigação e tratamento antes que complicaçōes maiores se manifestem.

A incidência de $19,5 \%$ de reoperaçōes é similar a outras séries e considerada aceitável para o período estudado. O achado mais comum nas reoperaçōes foi retração das cúspides associada a dilataçāo anular. Algumas vezes é possível realizar novo reparo, embora, em vários casos desta série, o cirurgiāo tenha preferido a troca valvar. Estenose mitral presente em 2 casos reoperados deve ter sido devida à evoluçāo do processo reumático.

Disfunçāo de prótese aórtica nāo associada ao reparo mitral foi causa de reoperaçāo.

A complicaçāo non-fatal mais comum foi embolia sistêmica em 9 casos, 3 dos quais tinham prótese aórtica e usavam anticoagulantes; isso poderia ser a causa de alguns óbitos classificados como súbitos ou

\footnotetext{
* FANTINI,F. A.; DRUMOND, L.F.; GONTIJO FILHO,B.; VRANDECIC, M.O. P.; CASTRO, M. F.; PAULA E SILVA, J. A.; BARBOSA, J. T.; BRACARENSE, L. F. A.; OLIVEIRA, C. A. H.; GUTIERRES, C.; PEDROZA, A. A.; HORTA, M G. C. - Valvuloplastia mitral em pacientes jovens com cardiopatia reumática. Apresentado no $19^{\circ}$ Congresso Nacional de Cirurgia Cardíaca, Sảo Paulo, Maio-1992.
} 
KALIL, R. A. K.; LUCCHESE, F. A.; PRATES, P. R.; SANT'ANNA, J. R. M.; FAES, F. C.; PEREIRA, E. M. C.; NESRALLA, I. A. Anuloplastia sem suporte para tratamento da insuficiência mitral reumática. Rev. Bras. Cir. Cardiovasc., 7(3):186-193, 1992.

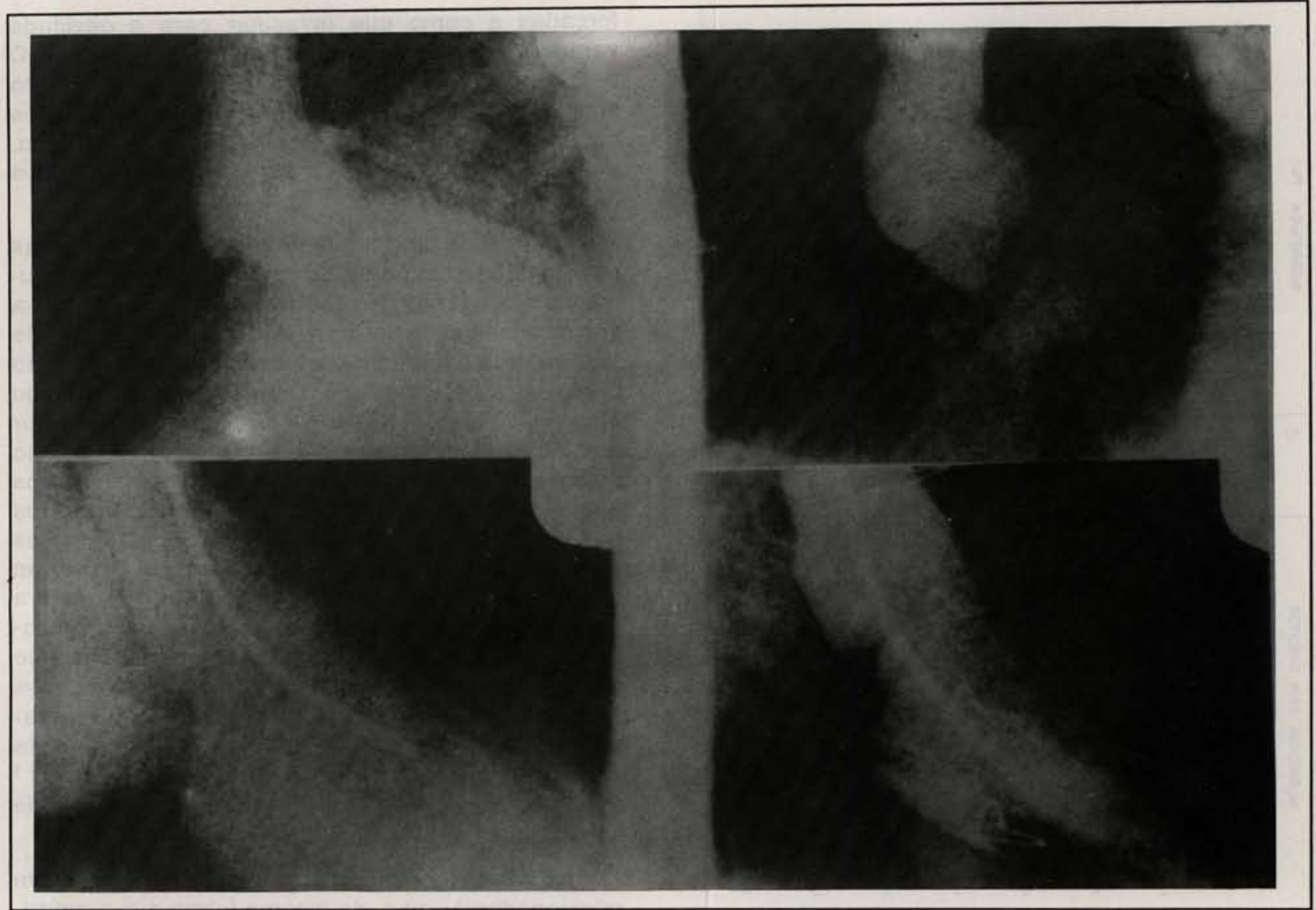

Fig. 5

de causa desconhecida. Os demais 6 pacientes apresentavam fibrilação atrial. Além de embolia pulmonar e sistêmica, houve a ocorrência de endocardite infecciosa, a qual poderá ser tratada como uso apropriado de antibióticos. Portanto, infecçāo em valvas reparadas pode ser tratada conservadoramente com sucesso, isso pela ausência de material implantado.

Temos notado que, pacientes jovens com insuficiência mitral isolada, usualmente apresentam grandes cúspides e flexíveis com leve ou moderada fibrose, geralmente sem calcificaçāo. Em poucos casos, as cordas estāo alongadas ou rotas, o que, no entanto, nāo compromete a viabilidade da correçāo. No início da série, em alguns pacientes permaneceram leves jatos de regurgitaçāo. Isto tem sido minimizado com o evoluir da experiência associado ao simples procedimento de encurtamento das cordas. Pacientes reumáticos mais idosos apresentam com maior freqüên- cia dupla lesāo mitral em valvas severamente fibrosadas e calcificadas. Duplas lesōes nāo foram incluídas neste trabalho, embora também passiveis de reparo, sendo que os pacientes mais idosos aqui incluídos nesta séria apresentavam apenas insuficiência mitral; nesta situaçāo, geralmente as valvas sāo flexíveis e a anuloplastia sem suporte pode ser empregada com sucesso.

Concluindo, a regurgitaçāo mitral reumática pode ser efetivamente tratada pela técnica da anuloplastia sem suporte, procedimento mais rápido e com excelentes resultados funcionais. A sobrevida a longo prazo é similar às técnicas mais complexas e evitam as complicaçōes dos implantes sintéticos ${ }^{8}$. O método é particularmente aplicável a crianças em fase de crescimento e pacientes do sexo feminino passíveis de engravidar, possibilitando sobrevida longa e sem prótese implantada. 
KALIL, R. A. K.; LUCCHESE, F. A.; PRATES, P. R.; SANT'ANNA, J. R. M.; FAES, F. C.; PEREIRA, E. M. C.; NESRALLA, I. A. - Non-supported mitral annuloplasty technique for treatment of rheumatic mitral insufficiency. Rev. Bras. Cir. Cardiovasc., 7(3):186-193, 1992.

ABSTRACT: Since 1974 a non-supported mitral annuloplasty technique has been employed for treatment of pure mitral regurgitation (PMR), in a population that was predominantly young and of rheumatic ethiology. An evaluation of late results forms the basis of this report. There were 154 patients operated on for PMR, 55 $(36 \%)$ male and $99(64 \%)$ female. Mean age was $36 \pm 16$ (5 to 73 ) years. Associated lesions were: 47 aortic 21 tricuspid and 2 ASDs (atrial aptal defects). Cases with concomitant mitral stenosis were not included. Properative functional class was $\mathrm{I}-\mathrm{II}$ in $19 \%$ and III-IV in $81 \%$. The cardiothoracic ratio was $0.61 \pm 0.10$. All patients were submitted to an unsupported mitral annuloplastic procedure, similar to that described by WOOLER, that consisted in reduction of the mural portion of the annulus obtained with the application of two buttressed mattress sutures at the comissures without compromisse to the width of the septal leaflet. When necessary, addictional chordal procedures were performed. No patients received ring or posterior annular support. Residual late systolic murmur was present in $48 \%$. Late complications were: systemic thromboembolism $5.8 \%$ ( $1 / 3$ with aortic prosthesis), infective endocardites $1.3 \%$ and pulmonary thromboembolism $0.7 \%$. Postoperative functional class was $\mathrm{I}-\mathrm{II}$ in $84 \%$ and $\mathrm{III}-\mathrm{IV}$ in $16 \%$. Cardiothoracic ratio was $0.58 \pm 0.10$. Actuarial probability of late survival was $79.5 \pm 5.3 \%$ at 10 years and $71.0 \pm 7.4 \%$ at 14 years. Event free survival was $67.9 \pm 8.9 \%$ at 10 years and $56.1 \pm 11.7 \%$ at 14 years. Rheumatic mitral regurgitation can be effectively treated by annuloplasty without prosthetic annular support with late results comparable to those obtained with more complicated procedures. This is particularly important for treatment of children and young patients, specially females at child-bearing age which, in some areas, form a substantial cohort in mitral disease.

DESCRIPTORS: mitral valve, surgery; valvuloplasty; rheumatic mitral regurgitation.

AGRADECIMENTO: Os autores agradecem a colaboraçāo da Dra. Marinez Barra Rossi, Dr. Luís Angarita Navarro, Doutorando René Jacobsen e Sr. César Coelho Pinto, na elaboração e na coleta de dados deste trabalho.

\section{REFERÊNCIAS BIBLIOGRÁFICAS}

1 ANTUNES, M. J.; MAGALHÄES, M. P.; COLSEN, P. R.; KINSLEY, R. M. - Valvuloplasty for rheumatic mitral disease: a surgical challenge. J. Thorac. Cardiovasc. Surg., 94: 44-56, 1987.

ARRUDA, M. B.; MORAES, C. R.; LAGRECA, J. R.; ESCOBAR, M.; VICTOR, E. G.; CAVALCANTI, I. L.; RAPOSO, L.; GOMES, J.M. P.; SOUTO, M. -Anuloplastia mitral. Arq. Bras. Cardiol., 27 (Supl. 1): 32, 1974 (Resumo).

3 BASHOUR, T. T.; ANDREAE, G. E.; HANNA, E. S.; MASON, D. T. - Reparative operations for mitral valve incompentence: an emerging treatment of choice. Amer. Heart J., 113: 1199-1206, 1987.

BRAILE, D. M.; ARDITO, R. V.; PINTO, G. H.; SANTOS, J. L. V.; ZAIANTCHICK, M.; SOUZA, D. R. S.; THEVENARD, R. - Plástica mitral. Rev. Bras. Cir. Cardiovasc., 5: 86-98, 1990.
CARPENTIER, A.; CHAUVAUD, S.; FABIANI, J. N.; DELOCHE, A.; RELLAND, J.; LESSANA, A.; D'ALLAINES, D. L.; BLONDEAU, P. L.; PIWNICA, A.; DUBOST, C. L. - Reconstructive surgery of mitral valve incompetence: tem year appraisal. J. Thorac. Cardiovasc. Surg., 79: 338-348, 1980.

6 COOLEY, D. A.; FRAZIER, O. H.; NORMAN, J. C. - Mitral leaflet prolapse: surgical treatment using a posterior annular collar prosthesis. Cardiovasc. Dis. Bull. Texas Heart Inst., 3: 438, 1976.

7 COSGROVE, D. M.; CHAVEZ, A. M.; LYTLE, B. W. Results of mitral valve reconstruction. Circulation, 74 (Parte 2): 182-187, 1986.

8 DAVID, T. E.; KOMEDA, M.; POLLICK, C.; BURNS, R. J. - Mitral valve annuloplasty: the effect of the type on left ventricular function. Ann. Thorac. Surg., 47: 524$528,1989$.

9 DELOCHE, A.; JEBARA, V. A.; RELLAND, J. Y. M.; CHAUVAUD, S.; FABIANI, J.; PERIER, P.; DREYFUS, G.; MIHAILEANU, S.; CARPENTIER, A. - Valve repair with Carpentier techniques: the second decade. J. Thorac. Cardiovasc. Surg., 99: 990-1002, 1990.

DURAN, C. G.; POMAR, J. L.; REVUELTA, J. M.; GALLO, I.; POVEDA, J.; OCHOTECO, A.; UBAGO, J. L. Conservative operation for mitral insuficiency: critical analysis supported by postoperative hemodynamic 
KALIL, R. A. K.; LUCCHESE, F. A.; PRATES, P. R.; SANT'ANNA, J. R. M.; FAES, F. C.; PEREIRA, E. M. C.; NESRALLA, I. A. Anuloplastia sem suporte para tratamento da insuficiência mitral reumática. Rev. Bras. Cir. Cardiovasc., 7(3):186-193, 1992.

studies of 72 patients. J. Thorac. Cardiovasc. Surg., 79: 326-337, 1980.

KALIL, R. A. K.; PRATES, P. R.; LUCCHESE, F. A.; BERTOLETTI, V. E.; PEREIRA, E. M.; SANT'ANNA, J. R. M.; NESRALLA, I. A. - Valvoplastia para insuficiência mitral: técnicas e resultados. Arq. Bras. Cardiol., 37: 315-319, 1981.

KALIL, R. A. K.; SANT'ANNA, J. R. M.; LUCCHESE, F. A.; PRATES, P. R.; VITOLA, D.; GARCIA-RAMIREZ, M.; PEREIRA, E.; NESRALLA, I. A. - Long. term results of annuloplasty for mitral regurgitation. J. Cardiov. Surg., 31: 14-19, 1990.

KALIL, R. A. K.; VITOLA, D.; FALLEIRO, R.; FELDMAN, C. J.; SANTANNA, J. R. M.; PRATES, P.R.; LUCCHESE, F. A.; PEREIRA, E. M.; COSTA, A. R.; LARA, R. F.; DAUDT, N. S.; NESRALLA, I. A.; RODRIGUES, R. Valorização da valvoplastia na correçāo da insuficiência mitral. Rev. AMRIGS (Porto Alegre), 33: 14-18, 1989.

WOOLER, G. H.; NIXON, P. G. F.; GRIMSHAW, V. A.; WATSON, D. A. - Experience with the repair of the mitral valve in mitral incompetence. Thorax, 17: 49, 1962.

\section{Discussāo}

\section{DR. LUIZ EDUARDO V. LEĀO São Paulo, SP}

Gostaria de agradecerà Comissāo Organizadora - privilégio de comentar a importante contribuição aqui propiciada pelo $\mathrm{Dr}$. Kalil e co-autores, onde demonstraram excelentes resultados tardios com a anuloplastia mitral pela técnica de Wooler, sem utilizar anel protético. No nosso grupo, o Dr. Succi e eu, no Hospital do Coraçāo e no Hospital Bandeirantes, estamos também realizando a plástia no Hospital Bandeirantes, estamos também realizando a plastia da valva mitral com técnica sem suporte. Em casos de insuficiência mitral em crianças, adolescentes e muIheres jovens, temos empregado a técnica proposta por Reed, onde é feita anuloplastia por "medida padronizada" e com "hipercorreção" - a valva mitral é tornada menor que o normal, sem ficar estenótica. Em adultos, a valva fica com circunferência de $6 \mathrm{~cm}$, área de cerca de $2,85 \mathrm{~cm}^{2}$; em crianças a área é tornada proporcionalmente menor. O procedimento é rápido, simples e falcimente reprodutível, e o anel pode crescer. Penso que mais importante que demonstrar a superioridade de uma técnica sobre outra, o importante é reiterar veementemente o conceito de preservaçāo da valva mitral. O Dr. Kalil e seu grupo vêm trabalhando há muitos anos na conservação da valva mitral, como outros colegas no nosso meio, como Gregori, Pomerantzeff, Brofman, Jatene, Braile, Dobrianskij e vários outros. Os excelentes resultados obtidos com técnicas diferentes sugerem fortemente que todo cirurgiāo tem que conhecer o princípio e dominar uma técnica de conservaçāo. Isto é particularmente importante no nosso meio, com tantos reumáticos jovens. Os dados do Dr. Kalil, com até 14 anos de pós-operatório, comparam-se favoravelmente com qualquer prótese, mesmo em populaçāo de primeiro mundo. Outro ponto que gostaria de comentar é que quando nos vemos frente a uma casuística de plastia mitral, entendo que lá estão inseridos pelo menos três subgrupos: a) pacientes com insuficiência mitral pura em crianças e jovens, etiologia reumática b) pacientes mais idosos, com valva com degeneração mixomatosa c) pacientes com disfunçăo mais complexa, restrição à movimentaçāo das cúspides, geralmente dupla disfunçăo. Creio que no grupo $A$ todo cirurgiāo deve fazer a plastia valvar. Nos grupos $\mathrm{Be}$, a maioria dos grupos parece mostrar vantagem dos procedimentos conservadores, porém utilizando técnicas mais complexas. Perguntaria ao Dr. Kalil: 1. Como tem calculado a área valvar em criança? 2. Considera também a técnica de Wooler em pacientes com valva mixomatosa e naqueles pacientes em que há necessidade de associar-se descalcificaçāo, comissurotomia e papilarotomia, fenestraçōes das cúspides etc.? Muito obrigado. 
KALIL, R. A. K.; LUCCHESE, F. A.; PRATES, P. R.; SANT'ANNA, J. R. M.; FAES, F. C.; PEREIRA, E. M. C.; NESRALLA, I. A. Anuloplastia sem suporte para tratamento da insuficiência mitral reumática. Rev. Bras. Cir. Cardiovasc., 7(3):186-193, 1992.

DR. KALIL

\section{(Encerrando)}

Agradeço os comentários dos colegas. Em resposta às perguntas do $\mathrm{Dr}$. Leão, a área valvar, tanto em adultos como em crianças, tem sido calculada de acordo com a largura do folheto septal, ou seja, na aplicação dos pontos, é respeitada essa largura e este procedimento determinará a dimensăo do orifício final. Por outro lado, temos empregado a técnica em pacientes com degeneração mixomatosa, bem como após descalcificaçōes, com resultados satisfatórios. Muito obrigado. 